Horizons philosophiques

\title{
La philosophie latino-américaine : un procès territorial
}

\section{André Vidricaire}

Volume 2, numéro 1, automne 1991

De Buenos Aires à Québec

URI : https://id.erudit.org/iderudit/800886ar

DOI : https://doi.org/10.7202/800886ar

Aller au sommaire du numéro

\section{Éditeur(s)}

Collège Édouard-Montpetit

\section{ISSN}

1181-9227 (imprimé)

1920-2954 (numérique)

Découvrir la revue

\section{Citer cet article}

Vidricaire, A. (1991). La philosophie latino-américaine : un procès territorial. Horizons philosophiques, 2(1), 77-90. https://doi.org/10.7202/800886ar d'utilisation que vous pouvez consulter en ligne.

https://apropos.erudit.org/fr/usagers/politique-dutilisation/ 


\section{La philosophie latino-américaine : un procès territorial}

Québec, États-Unis, Argentine, Brésil, Mexique, Pérou, Équateur : qu'y a-t-il de commun entre ces pays qui partagent un même continent? Comment les regrouper, quand les États-Unis et le Canada sont deux des sept pays les plus industrialisés, tandis qu'en Amérique latine, d'autres vivent dans des conditions similaires à celles du tiers monde? Étant donné cette diversité politique et économique, comment rapprocher des expériences dans la réflexion philosophique? S'agit-il d'une juxtaposition spatiale d'histoires philosophiques qui s'additionnent ou, au contraire, de démarches, certes multiples et divergentes, mais qui se trouvent à questionner le contexte historique où elles sont nées?

Ici, nous prendrons comme point d'observation l'histoire de la philosophie latino-américaine. Nous décrirons les diverses étapes de son histoire pour montrer, à partir du concept de territorialité 1 , que cette expérience dans la réflexion philosophique, loin de se réduire à une imitation servile des philosophies européennes, est un questionnement de l'être latino-américain.

1. Je ne saurais trop souligner ici que cette problématique m'a été suggérée par Robert Hébert qui, depuis plus de 15 ans, poursuit une recherche extrêmement stimulante sur l'histoire des expériences québécoises en philosophie. 


\section{La scolastique ibérique de la période coloniale}

L'Amérique a été un lieu d'exercice de pouvoir que des hommes se sont attribué, il y a 500 ans. Résultat de conquêtes européennes antagonistes, la "découverte" de ce continent est vite devenue, au gré des visées et des stratégies divergentes des Espagnols, des Portugais, des Anglais et des Français, un ensemble hétérogène de productions territoriales faites de frontières, de points de repère et de réseaux de communication propres à chacun d'eux. Pur produit d'un centre qui a pour nom l'Europe, la formation de l'Amérique a consisté en l'implantation de divers systèmes territoriaux qui correspondaient aux motivations des hommes de l'Ancien Monde désireux de fonder une Nouvelle Angleterre, une Nouvelle France, une Nouvelle Espagne, une Nouvelle Grenade.

En Amérique latine, la pensée philosophique a débuté avec cette conquête de l'Amérique et en partagea les visées, les idées et les valeurs. Terre utopique sans passé ni tradition, la mère-patrie a imposé son canal de communication et l'usage exclusif de la scolastique ibérique. En effet, de même qu'elle a imposé une structure économicopolitique, de même, elle a édicté une géographie de la pensée, un espace dans la réflexion et donc des frontières qui ont déterminé la manière dont il fallait penser ce Nouveau Monde et en ce Nouveau Monde. Par exemple, seule philosophie valorisée et autorisée, cette forme de rationalité a fourni les fondements et les justifications au sujet de la nature, des droits et de la place des aborigènes. Ainsi, Quevedo, Sepulveda et Las Casas ont parlé de différence de nature ou de différence de degré entre les Indigènes et les Blancs pour en déduire, selon le cas, des droits différents. En effet, en vertu du principe aristotélicien que le moins parfait est soumis au plus parfait, il en résulte que les Indiens servent et obéissent aux Espagnols qui règnent et commandent.

Mais comment justifier la légitimité de la souveraineté du conquérant? Les Indiens n'étaient-ils pas les souverains 
de leurs terres et ce, par droit naturel et droit divin? Voici deux thèses. Partant des bulles d'Alexandre VI qui attribuent les découvertes aux Espagnols, Rubio justifie ce legs par le fait que Dieu a donné la souveraineté et la juridiction de la terre à Saint-Pierre et que ses successeurs en ont fait donation d'une partie au roi très catholique de l'Espagne. II en résulte que si les Indiens se font catholiques, ils seront traités comme des vassaux avec les droits afférents. S'ils refusent, le roi a le droit de les assaillir et de spolier leurs biens. De son côté, De Vitoria affirme que les Indiens sont souverains de leurs terres et que la donation du pape ne peut annuler ce droit puisque son pouvoir n'est que spirituel. Par contre, entrant en commerce avec les Indiens, si ces derniers respectent les droits d'échange et d'appropriation, les Espagnols doivent leur reconnaître les mêmes droits. Mais si les Indiens les en empêchent, les Espagnols sont justifiés de recourir à la force, voire de s'approprier leur territoire pour faire respecter ces droits. En résumé, les indigènes devaient s'insérer dans un système étatique patrimonial fondé sur un messianisme ibérique s'ils voulaient survivre.

\section{Le romantisme du $\mathrm{XIX}^{\mathrm{e}}$ siècle}

Au début du XIXe siècle, des groupes oligarchiques créoles, à l'écart de ce pouvoir, ont questionné cette Amérique portugaise et cette Amérique espagnole. Européens de droit mais Américains de naissance, au dire de Bolivar, ils découvraient qu'ils étaient une espèce différente de l'Europe et des Indiens. Questionnant leur identité, ils critiquaient la forme donnée à leur territoire tant sur les plans politico-économique que socio-culturel. Cherchant une structuration différente de cette Amérique où ils étaient nés, les exemples des révolutions américaine et française en montraient la faisabilité, tandis que la philosophie des Lumières et les philosophies romantiques élargissaient cette émancipation aux domaines intellectuel et religieux. Aussi, ont-ils troqué leur filiation obligée à la doctrine phi- 
losophique normative de la mère-patrie pour d'autres systèmes de pensée européenne.

\section{Le positivisme du XIXe siècle}

Mais ces visions de libération coloniale et d'émancipation politique, économique et intellectuelle, n'ont pas produit les fruits escomptés. Certes, le pouvoir monarchique était renversé. De plus, l'ordre social basé sur la religion était remplacé par un pacte social laïc basé sur la liberté et l'égalité de tous. Mais dans les faits, l'état était oligarchique et une nouvelle classe latifundiste dominait. Aussi, paraphrasant Octavio Paz, nous dirions que le révolutionnaire s'est fait réformiste libéral. II visait à corriger les abus du pouvoir - non le pouvoir lui-même - les excès du désordre - non l'ordre lui-même. Comme le premier, il croyait au progrès continu et en la raison capable de produire des changements définitifs où le passé ne revient pas et où le futur est cette perfectibilité indéfinie de l'espèce humaine. Mais le réformiste était un philosophe positiviste qui aborde les problèmes politiques et sociaux comme ceux de la nature. Une société est évolutive : elle a ses étapes, elle a ses lois. L'erreur a été d'accorder la liberté à des hommes alors que les conditions matérielles, sociales et politiques n'étaient pas réunies. Certes, le triomphe des forces libérales sur les forces négatives du clergé et des coloniaux est un gain énorme. À la suite de la loi des trois États de Comte, Baruda voit dans cette lutte, le passage du stade théologique au stade métaphysique. Mais le but de la révolution libérale est atteint : doit apparaître maintenant le stade positiviste où l'ordre politique et économique va guider les libertés individuelles. D'autre part, suite à Spencer et à Darwin, le principe d'égalité a été remplacé par la théorie de la lutte pour la vie, justifiant ainsi les hiérarchies et les fonctions, voire même le sacrifice de certains droits politiques pour développer l'industrie et le commerce. 


\section{Les philosophies au $X X^{e}$ siècle}

Le $X X^{\theta}$ siècle a connu des révolutions qui mettent en cause l'intériorité des états et leur identité. En concomitance avec le renouvellement des structures politiques, des critiques ont surgi au sujet de la capacité du positivisme à fournir une orientation à l'existence humaine et à ces peuples. Rodo a remis en question l'idée que le scientisme soit le seul indicateur des voies éthique, politique et esthétique de l'homme. En 1900, présentant la figure symbolique d'Ariel comme l'épitomé de tout ce qui est spirituel, Rodo l'a opposé à Caliban de nature plus utilitariste. Comme les qualités spiritualistes ont été fortement cultivées par les nations latines de l'Europe, Rodo a proposé aux Latins du Nouveau Monde d'être les légataires de ces qualités ariélistes, se distinguant ainsi des États-Unis qui connaissaient, certes, une prospérité matérielle extraordinaire, mais qui manifestaient, par ailleurs, "une incompréhension des valeurs humaines et de la destinée humaine»:

Manquant d'orientation en lien avec des traditions établies, cette nation n'a pas découvert comment remplacer l'idéalisme du passé par une vision du futur élevée et désintéressée.

Des intellectuels dont José Vasconcelos (1882-1959), Antonio Caso (1883-1946), Alfonso Reyes (1889-1959) qui ont fondé en 1910 l'Athénée des jeunes ont poursuivi cette approche spiritualiste. Ce groupe a partagé en partie la critique ariéliste du scientisme, en réaffirmant des valeurs autres que le matérialisme issu d'un positivisme sans âme. Ce qui est à la base des empires et des peuples, c'est l'énergie vitale et non pas les déterminismes historiques et sociaux tels que définis par Comte. Caso a développé l'idée d'une détermination individuelle de la conscience, tandis que Vasconcelos dans La race cosmique (1925) et Indologia (1926) a traité de la grande race cosmique du futur en Amérique du Sud, issue de l'amalgame des races de l'Espagne, de la Grèce, du Mexique et des Indes. Ainsi, 
au lieu d'une identité issue de la latinité européenne, on a pour identité sa propre géographie. Ricardo Rojas (18821957) a montré dans Eurindia (1924) que la race n'est pas seulement une matière d'éthos scientifique, mais aussi une conscience collective de sens qui s'affirme sur une émotion territoriale et sur une expérience commune. En résumé, très proche du romantisme idéaliste, cette doctrine a reconnu à ce continent une essence et un esprit propres qui s'expriment en une civilisation originale.

Mais alors, comment expliquer que l'Amérique du Sud ne soit pas arrivée à réaliser sa destinée spirituelle? Samuel Ramos (1897-1959) et Octavio Paz ont procédé à un examen de personnalité - manque de confiance, agressivité, ressentiment - qui conduit à des comportements de machisme et de dissimulation; ils ont montré que ce complexe d'infériorité explique les déficiences culturelles dont celle de la prédominance à l'imitation et au dédoublement.

Par ailleurs, cette analyse psychoculturelle des déficiences a conduit à une réflexion sur les empêchements et les impossibilités issus de situations de dépendance, de domination, d'oppression et d'injustice dans une Amérique colonisée. Cette problématique a donné lieu à une révision du passé, notamment en histoire des idées et à l'élaboration d'une philosophie pour la libération de tous les hommes basée sur l'humanisation, la conscientisation et la solidarité.

\section{Quatre procès territoriaux}

En résumé, l'histoire politique de ces pays d'Amérique latine est l'histoire d'une dépendance avec tous ses avatars : 1) le régime colonial qui dure presque trois siècles; puis 2) les guerres d'indépendance entre 1800-1825. Ensuite 3), l'état libéral de type dictatorial jusqu'aux grandes révolutions nationales 4 ) en ce $X X^{\ominus}$ siècle. À ces quatre moments de cette histoire économico-politique correspondent les pratiques philosophiques suivantes : 1) philoso- 
phie scolastique; 2) philosophie des Lumières et philosophies romantiques; 3) philosophies positivistes; 4) tous les courants philosophiques contemporains à côté de la philosophie pour la libération. Comme ces étapes de la pensée correspondent directement aux grands courants philosophiques européens, il s'en faut de peu pour conclure qu'il s'agit d'une tragique histoire d'une réflexion latino-américaine en philosophie qui se réduit à une répétition et à une imitation sans fin de la philosophie européenne. À cause d'une dépendance d'abord politico-économique, puis culturelle, cette pensée n'aurait fait que suivre des modes européennes et reproduire des philosophies qui se pratiquaient ailleurs. Dans ces pays, il n'y aurait pas eu de philosophie originale; dans ces pays, il n'y aurait pas eu de figures créatrices qui auraient fondé et suscité une tradition qui leur serait propre. Tout commencerait maintenant.

Cette interprétation évaluative de l'histoire de la philosophie latino-américaine me semble une erreur. On peut en faire une autre lecture. On peut la décrire et la comprendre autrement si nous analysons, à partir du concept de territorialité, ces quatre moments qui scandent l'histoire politique et philosophique de l'Amérique latine.

I. La territorialité est un rapport avec les êtres et les choses qui peut prendre diverses formes, eu égard aux frontières et aux voisinages établis. Ainsi, la colonisation n'est pas un simple élargissement d'un espace déjà structuré, mais plutôt une restructuration en nouvelles frontières et en nouveaux réseaux de voisinage qui transforment de fond en comble l'espace initial. Par exemple, l'Espagne et le Portugal d'avant les conquêtes voient l'organisation de leur propre territoire complètement changée suite à l'appropriation de ce nouvel espace en Amérique. En effet, dans cette nouvelle totalité, ces pays européens mettent en place un nouveau "procès territorial» où ils sont le centre de fonctions politique, économique et culturelle sur d'autres lieux situés en périphérie. Cette nouvelle forme de ter- 
ritorialité a pour effet de réorganiser leurs pratiques de pouvoirs et de savoirs. En effet, ces pays européens deviennent des capitales externes de décision, de contrôle et de ratification de délégation de pouvoirs et de savoirs qui s'exercent tant en leur centre qu'en périphérie. Ainsi, en philosophie, ces capitales édictent que la scolastique ibérique est la seule doctrine en usage en Amérique. Mais cette philosophie colonialiste et endocolonisante n'est déjà plus la copie exacte de la norme imposée. En effet, outre le simple commentaire de la doctrine - la plupart du temps enseignée en latin - , des philosophes de la périphérie ont réfléchi sur des thèmes américains jusqu'alors absents de cette doctrine. Certes, cette réflexion se fait selon le projet, la perspective et le point de vue de l'Europe; le mode de réflexion utilisé est celui-là même en usage en Occident. Néanmoins, il faut bien constater que c'est la première fois que des intellectuels de ce Nouveau Monde, en portant leur réflexion sur leur propre réalité, abordent des questions qui élargissent la thématique européenne et qui suscitent des polémiques tant dans leur propre territoire que dans la mère-patrie. Évidemment, ce sont les idées les plus appropriées aux visées du colonisateur qui ont triomphé et ce sont ces mêmes idées qui, au dire de Paz, ont mutilé l'être latino-américain. L'intérêt est d'apercevoir que cette vision dominante implique une lutte qui a réduit au silence d'autres visions et qui a banni celles qui venaient la contredire.

II. La création de ces nouveaux espaces de pouvoirs et de savoirs en centre et périphérie a instauré, en chacun de ces lieux, une temporalité propre qui n'a pas été nécessairement convergente. Ainsi se sont manifestées des différenciations, puis des discontinuités. Le passage du colonialisme à l'indépendance, au début du XIX $X^{\ominus}$ siècle, en est une première illustration. En effet, l'Espagne et le Portugal ont cessé d'être des capitales : les pays latinoaméricains, s'arrachant du sol européen, sont devenus à la fois des centres de décision et des fragments de l'éthos 
féodal ibérique qui a connu un développement original. En effet, selon Hartz' ${ }^{2}$, «la logique du processus de fragmentation" a pour caractéristique que "les idéologies européennes une fois transplantées hors du champ de bataille originel, séparées du processus de renouvellement qui fait que la lutte se poursuit sans cesse, connaissent l'épanouissement de leurs possibilités latentes étouffées en Europe". C'est ce qui est survenu dans la pensée latino-américaine : d'un côté, elle a rompu avec l'Europe qui lui a donné naissance, de l'autre, elle s'est coupée des doctrines futures qui se sont affrontées en Europe. Mais, par ailleurs, elle a réalisé et accompli pour son territoire toutes les virtualités d'une vision du monde traditionnelle. Cela ne signifie pas que l'Amérique ne soit pas modernisée. Au contraire, la fragmentation montre plutôt que son processus a eu pour conséquence qu'en ce $\mathrm{XIX}^{\ominus}$ siècle les philosophies romantique et positiviste ont été des systèmes de pensée pour adapter au temps et à la conjoncture un ordre qui demeure traditionnel. En un mot, ces philosophies ont servi à défendre et à justifier ou encore à critiquer et à réformer des savoirs et des pouvoirs qui devaient être conformes à cet ordre. Par ailleurs, cet usage de pensées exogènes aboutit à des développements qui ne se trouvaient pas dans le modèle européen.

III. Mais il faut les événements du XX ${ }^{\ominus}$ siècle — forte émigration de populations non ibériques, révolutions nationales, guerre d'Espagne et deuxième Guerre mondiale, crise de la culture occidentale - pour que la pensée traditionnelle soit remise en question et qu'émerge une critique du territoire comme territoire périphérique et fragment.

2. La théorie de la "fragmentation" est développée par Louis Hartz (Les enfants de l'Europe, Paris, Seuil, 1968) qui, par son approche diachronique, me semble apporter un éclairage fécond à la théorie du "centre - périphérie» développée par Raffestin. Robert Hébert consacre une note à Hartz dans son livre l'Amérique française devant l'opinion étrangère 1756 - 1960, Montréal, l'Hexagone, 1989. 
En effet, au dire de Zea ${ }^{3}$, quand l'homme latino-américain qui avait vécu confiant sous l'arbre occidental qu'il n'avait pas planté, a découvert que cet arbre était en crise, il en a éprouvé un grand désarroi qui demeure encore : en effet, il s'est vu dans l'obligation de planter son propre arbre culturel et de créer ses propres idées. Mais comment cela pouvait-il se réaliser quand l'Amérique du Sud est néanmoins une branche de l'Europe et que la crise qui se produit là-bas est aussi sa crise?

En philosophie, cette question existentielle a pris de multiples directions. À côté des pratiques qui ont visé à s'inscrire dans de grandes orientations philosophiques internationales - Bunge, Unger, Castaneda, etc. -, s'est développée une approche historiciste dont les développements ont débouché sur une philosophie pour la libération. En premier lieu, ce courant a favorisé l'étude systématique de l'histoire des idées latino-américaines : cette étude devait aboutir à une critique des traumatismes dans la pensée, des incapacités et des inadaptations issues d'un sentiment d'infériorité et d'une dépendance vis-à-vis de l'Europe. En second lieu, cette élucidation critique des expériences dans la réflexion philosophique a donné lieu à un questionnement proprement philosophique sur de multiples problèmes posés par cette "circonstance» historique qui étaient soit inconnus de l'Europe soit non formulés dans les mêmes termes. II en est résulté des thèses sur la dépendance, l'identité, la culture, l'histoire et finalement l'affirmation de la possibilité de la libération de l'homme dans une culture universelle différenciée.

\section{En guise de conclusion}

L'histoire des pays latino-américains du point de vue de leur territorialité comporte quatre moments auxquels correspondent quatre structurations différentes des pou-

3. L. Zea, "Essays on Philosophy in History" dans Latin American Philosophy, éd. par Jorge J.E. Gracia. 
voirs et des savoirs. Ainsi, la philosophie latino-américaine débute dès la période coloniale et ce, malgré l'absence d'appareils sophistiqués pour philosopher. Mais comme cette réalité américaine se pense dans les termes de la philosophie européenne, faudra-t-il dire que la pensée qui en résulte est une image illusoire de celle-ci? S'agit-il d'une projection du regard de l'Europe sur ce nouveau continent qui a pour effet de créer une représentation mystifiée et irréelle de ce dernier? Pour que les intéressés soient euxmêmes capables de poser cette question et d'y répondre, je dirais qu'ils doivent avoir expérimenté diverses formes de rencontre avec l'Europe comme diverses formes de découverte de leur univers. Nous le savons : l'identité d'un individu, d'un groupe ou d'un peuple comme différente et égale émerge de son rapport avec le monde et avec autrui. C'est le vécu, l'itinéraire, voire le parcours historique de ce rapport, certes conditionné par les diverses structurations, qui a conduit les Latino-Américains à prendre conscience de leur identité, à la connaître, à l'approfondir et finalement, de période en période, à en tracer une manière de se vivre qui soit liée à un passé et à un projet collectif d'avenir. Cette révision critique de l'identité et son dépassement se sont produits aussi bien au niveau des savoirs que des pouvoirs. Par exemple, c'est à la suite des diverses expériences dans la pensée philosophique aux époques coloniale et post coloniale, que les philosophes du $X X^{e}$ siècle ont proposé d'en écrire et les histoires nationales et l'histoire continentale. Cette histoire des idées ne cherche pas à nier les déficiences de cette expérience en philosophie, mais bien à se demander par exemple "comment, pourquoi et dans quels buts les philosophies exogènes ont été adoptées". Ainsi, cette histoire permet de révéler qu'il y a eu des usages des philosophies européennes complètement étrangers à leur propre contexte. De même, elle montre que des pratiques philosophiques locales ont eu pour fonction "de sanctionner un niveau de conscience en accord avec des modèles de culture qui n'avaient aucune racine» 
avec les conditions réelles d'existence. Encore une fois, il ne s'agit pas de nier ces faits, mais de dépasser leur dénigrement en les abordant comme des expériences singulières dans la réflexion philosophique.

Du point de vue de la territorialité, on découvre alors que cette histoire n'est pas une quête d'identité qui aurait pour point de départ un «je ne suis rien» à combler. Elle débute plutôt par un excès de sens donné par l'Europe qui a projeté en terre d'Amérique ses propres utopies. Puis, lors de la fragmentation, le bourgeon a accompli un legs. On le voit : l'Europe a servi de médiation symbolique pour l'identification de l'Amérique du Sud pendant plusieurs siècles. Paz écrit : "Toute l'histoire du Mexique, depuis la Conquête jusqu'à la Révolution, peut être considérée comme une recherche de nous-mêmes, déformés et masqués par des institutions étrangères, et d'une forme qui nous exprime».

Il en est résulté des expériences d'inauthenticité dans la réflexion : cela va de soi. Mais il y a eu aussi des pensées authentiques en ce contexte terriblement inauthenti$q^{4}{ }^{4}$. II y a eu aussi des silences, des peurs, des colères. En effet, étant le wagon du train occidental, les LatinoAméricains ont découvert que leur libération consistait à tracer leur propre voie avec leurs rails pour faire de leur wagon leur propre train 5 . Or, cette histoire est possible parce qu'elle reprend ce que les précurseurs ont commencé... sur ce même territoire appelé à se transformer.

\section{ANDRÉ VIDRICAIRE Université du Québec à Montréal}

4. Le philosophe Zea a des pages admirables sur cette question.

5. Je reprends ici l'image utilisée par Enrique Dussel. 


\section{Bibliographie}

Ardao, A. "Assimilation and Transformation of Positivism in Latin America", Journal of the History of Ideas. $N^{\circ} 24$ (1963) p. 515-522.

Bondy, Salazar, A. The meaning and Problem of Hispanic American Philosophic Thought., traduction anglaise. University of Kansas, 1969.

Cerutti-Guldberg, H. "Actual situation and perspectives of Latin American Philosophy for liberation", The Philosophical Forum, XX. Nos 1-2 (1988-1989) p. 43-61.

Dussel, Enrique. Histoire et Théologie de la Libération. Paris : éd. Ouvrière, 1974.

Flower, E. "The Mexican Revolt Against Positivism", Journal of the History of Ideas. $\mathrm{N}^{\circ} 10$ (1949) p. 115-129.

Gracia, Jorge J. "Importance of the History of Ideas in Latin America : Zea's. Positivism in Mexico", Journal of the History of ldeas. $\mathrm{N}^{\circ} 36$ (1975) p. 177-184.

Gracia, Jorge J.E. et Mireya, Camurati éd. Philosophy and Literature in Latin America. U.S.A. : Suny Press, 1989.

Gracia L. Jorge J.E. éd. Latin American Philosophy. U.S.A., 1986.

Hartz, Louis. Les Enfants de l'Europe. Paris : Seuil, 1968. Hébert, Robert. L'Amérique Française devant l'Opinion Étrangère 1756 - 1960. Montréal : Hexagone, 1989.

Maros Trends in Mexican Philosophy. U.S.A., 1966.

Marti, Oscar R. "ls There a Latin American Philosophy", Metaphilosophy. $\mathrm{N}^{\circ} 14$ (1983) p. 46-52.

Miro Quesada, F. "The Impact of Metaphysics on Latin American Ideology", Journal of the History of Ideas. $\mathrm{N}^{\circ} 24$ (1963) p. 539-552. 
Paz, Octavio. Le Labyrinthe de la Solitude. Paris : Gallimard, 1972.

Raffestin, Claude. Pour une Géographie du Pouvoir. Paris, 1980.

Rowanell, P. "Ortega in Mexico : a tribute to Samuel Ramos", Journal of the History of Ideas. $N^{\circ} 21$ (1960) p. 600-608.

Stabb, Martin S. In Quest of Identity. U.S.A., 1967.

Zea, Leopoldo. «Identity : a latin american philosophical problem", The Philosophical Forum, XX. Nos 1-2 (1988-1989) p. 33-42. 